\title{
Rise of Systems Glycobiology and Personalized Glycomedicine: Why and How to Integrate Glycomics with Multiomics Science?
}

\author{
Tanja Kunej
}

\begin{abstract}
Glycomics is a rapidly emerging subspecialty of system sciences that evaluates the structures and functions of glycans in biological systems. Moreover, glycomics informs allied scholarships such as systems glycobiology and personalized glycomedicine that collectively aim to explain the role of glycans in person-to-person and between-population variations in disease susceptibility and response to health interventions such as drugs, nutrition, and vaccines. For glycomics to make greater, systems-scale, contributions to biology and medical research, it is facing a new developmental challenge: transition from single omics to multiomics integrative technology platforms. A comprehensive map of all possible connections between glycomics and other omics types has not yet been developed. Glycomics aims to discover a complex interplay of molecular interactions; however, little is known about the regulatory networks controlling these complex processes. In addition, the glycomics knowledgebase is presently scattered across various publications and databases, and therefore does not enable a holistic or systems view of this study field. Therefore, researchers are not always aware, for example, that a given analyzed genetic locus is linked with glycans, and that there are also glycomics determinants of complex phenotypes in health and biology. This review presents several published examples of glycomics science in association with other omics levels, such as genomics, transcriptomics, proteomics, metabolomics, epigenomics, ncRNomics, lipidomics, and interactomics. I also highlight the salient knowledge gaps and suggest future research directions. Understanding the interconnections of glycomics with other omics technologies will facilitate multiomics science and knowledge integration, enhance development of systems glycobiology and personalized glycomedicine.
\end{abstract}

Keywords: systems glycobiology, glycome, glycomics, multiomics, personalized medicine, personalized glycomedicine

\section{Introduction}

G LYCOMICS IS ONE OF THE LATEST Omics system science fields that evaluates the structures and function of glycans in biological systems (Adua et al., 2017). While there have been early hints that integration of multiple omics fields with glycomics might offer new system-scale insights in integrative biology (Ly et al., 2010), a current discussion is warranted on the ways in which the emerging glycomics technologies can usefully be combined with other omics knowledge and technologies such as genomics, proteomics, metabolomics, and others yet to emerge in the near future (Fig. 1). Moreover, glycomics informs other crucial schol- arships such as systems glycobiology and personalized glycomedicine that collectively aim to explain the role of glycans in person-to-person and between-population variations in disease susceptibility and response to health interventions such as drugs, nutrition, and vaccines.

Glycan is a compound consisting of a large number of monosaccharides linked glycosidically (oligosaccharides or polysaccharide) or a carbohydrate portion of glycoconjugates, such as glycoprotein, glycolipid, or a proteoglycan. Glycome is the entire complement of glycans or glycoconjugates in a cell, tissue, or organism. Glycoside is a molecule in which a sugar is bound to another functional group (to another glycan or an aglycone) through a glycosidic

University of Ljubljana, Biotechnical Faculty, Department of Animal Science, Domzale, Slovenia. 


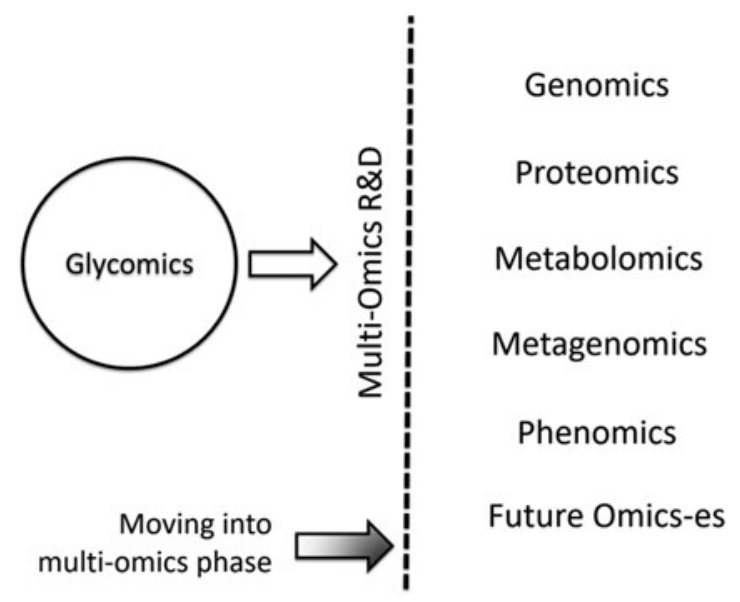

FIG. 1. Glycomics technologies could be combined with other omics knowledge and technologies such as genomics, proteomics, metabolomics, and other emerging omics types, therefore enabling a shift from single omics to multiomics approach.

bond. A glycosidic bond is a type of covalent bond that joins a carbohydrate (sugar) molecule to another group, which may or may not be another carbohydrate. Various glycosidic bonds are known: S-, N-, C-, and O-glycosidic bonds (Bennun et al., 2016; Reily et al., 2019).

Glycosylation is a form of cotranslational and posttranslational modification, and it is the enzyme-catalyzed covalent attachment of a carbohydrate to a polypeptide, lipid, polynucleotide, carbohydrate, or other organic compound, generally catalyzed by glycosyltransferases, utilizing specific sugar nucleotide donor substrates. Glycosyl transfer can also occur to protein residues, usually to tyrosine, serine, or threonine to give O-linked glycoproteins, or asparagine to give $\mathrm{N}$ linked glycoproteins. Glycoside hydrolases or glycosidases are enzymes that break glycosidic bonds (Reily et al., 2019).

Glycogenes (also termed glycan genes) are genes, involved in glycan biosynthetic pathways. For example, MGAT3 is a glycogene encoding for a glycosyltransferase mannosyl (beta1,4-)-glycoprotein beta-1,4-N-acetylglucosaminyltransferase. According to the latest release of the HGNC (HUGO gene nomenclature committee) database, there are 210 genes annotated within the gene group glycosyltransferases. They are classified into 26 subgroups and include genes such as $O G T$, MGAT5, and ST3GAL1 (https://www.genenames.org/data/ genegroup/\#!/group/424) (Yates et al., 2017). For example, OGT (O-linked N-acetylglucosamine (GlcNAc) transferase) is the enzyme driving O-GlcNAcylation.

Assembly of glycome structures requires a coordinated activity of various proteins, such as glycosyltransferases and glycosidases (Agarwal et al., 2015). Glycocode also termed a sugar code is a coding system on the basis of carbohydrates (Gabius et al., 2011). It has a large coding capacity since the theoretical number of oligomers formed by monosaccharides is much higher than that formed by using nucleotides or amino acids (Gabius and Kayser, 2014). Contrary to proteins, glycan biosynthesis does not require a template, and its structure is catalyzed by various enzymes that attach or detach monosaccharides in the glycosylation processes, therefore making glycomics research more challenging than proteomics or genomics (Adua et al., 2017). The variety and dynamic in glycome composition enables a quick response to environmental stimuli (Lauc et al., 2016).

Systems glycobiology is a subfield of systems biology, and is a holistic approach to reveal complex glycosylation process and relations among its components. It aims to provide a more complete glycome overview based on glycan annotation and also on other aspects, such as enzymatic levels, glycans abundances, biosynthetic pathways, and associated omics data sets. Systems glycobiology approach is based on combining multiple glycomics data sets with other omics data using glycoinformatics to elucidate insights on glycosylation process from multiple data sets (Bennun et al., 2016).

Glycosylation is involved in both normal physiology and disease development (Varki, 2017). Aberrant glycosylation is a feature of several diseases. Differential glycosylation patterns could distinguish between disease and health state, and serve as stratification biomarkers (glycobiomarkers) in personalized medicine (Reily et al., 2019). Glycobiology and glycopathology will therefore enable several new medical implications (Gabius and Kayser, 2014). Holistic insights into the structure and function of the glycome can be applied to therapy development, fine-tuning immunological responses and the performance of therapeutic antibodies and boosting immune responses, therefore greatly contributing to the development of the emerging field of glycomedicine (Reily et al., 2019).

Glycan arrays offer many applications in basic biochemical research to biomedical applications (Geissner and Seeberger, 2016). Shotgun glycan microarrays extended the defined glycan microarrays to a platform that supports functional glycomic analyses glycome (Smith et al., 2019). In addition, cell-based array resource has been developed, which displays the human glycome in a natural glycoconjugate and cell-surface context and will enable deeper understanding of the many biological roles of the glycome (Narimatsu et al., 2019). However, multiplatform and multiomics technologies are expected to further deepen our understanding of molecular mechanisms.

\section{Integrating Glycomics with Other Omics Technologies and Knowledge}

Systems glycobiology is a study field that aims to characterize cellular glycosylation processes based on integrating various omics data (Bennun et al., 2016). Integrating biomarkers across omics platforms has been shown to improve prediction accuracy. The integration of glycomics with other types of biomarkers offers further possibilities for innovation in diagnosis and treatment of diseases. For example, multivariate models consisting of different omics data (DNA methylation, transcriptomic, proteomic, and glycomic) obtained from analyses on different biomaterials have been shown to improve accuracy for prostate cancer risk stratification (Hart and Copeland, 2010; Murphy et al., 2018).

The first step toward classification of these complex genomics information according to omics levels was enabled by proposed taxonomy of multiomics science (Pirih and Kunej, 2017) and extended in previous studies (Pirih and Kunej, 2018; Redenšek et al., 2018) (Fig. 2). As other omics types, glycomics could be studied at a single locus or pathway, or at the whole genome level (Pirih and Kunej, 2018). Before multiomics data integration, data should first be sorted 


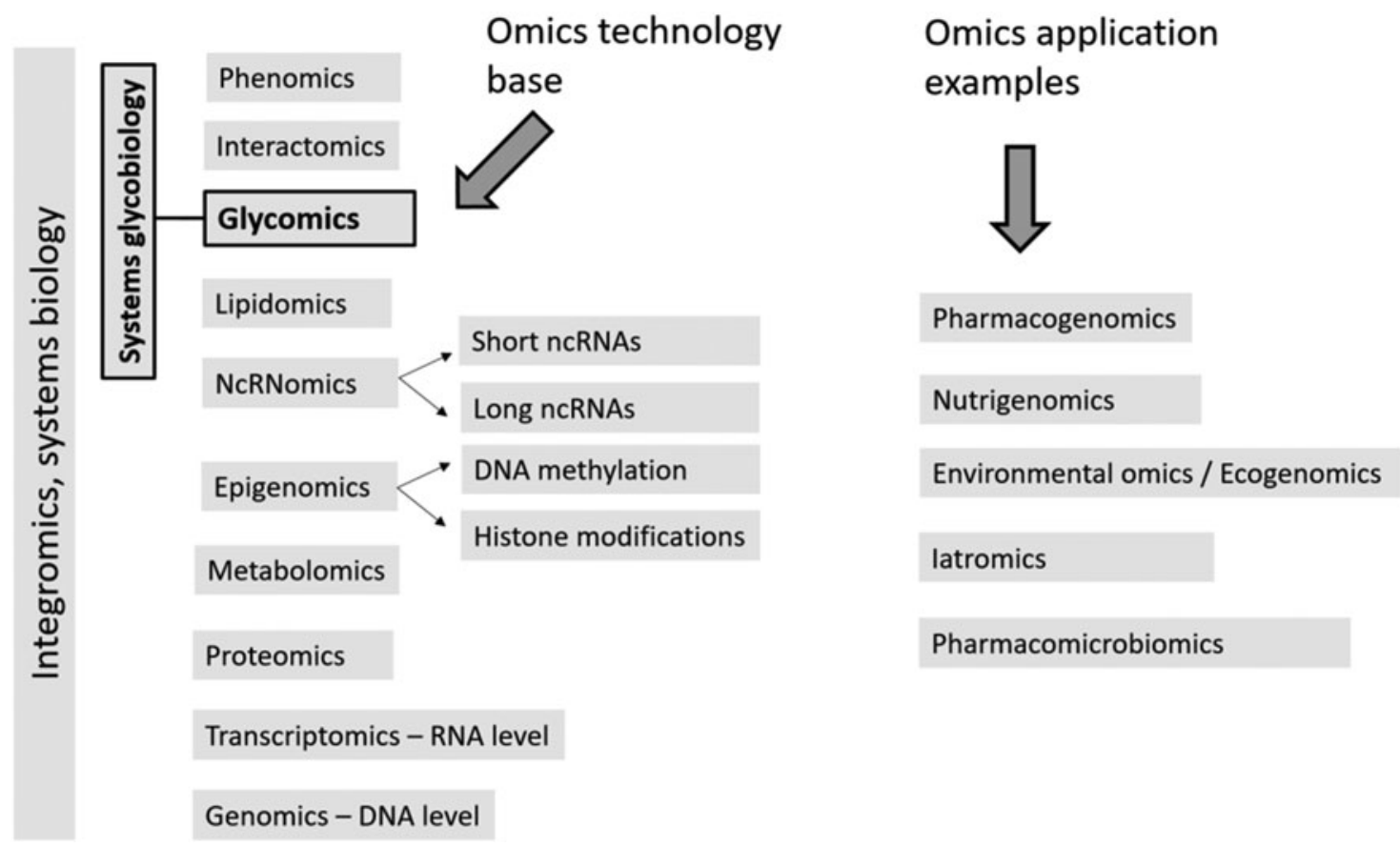

FIG. 2. Glycomics in the context of multiomics taxonomy.

according to the separate omics levels. The following chapter presents selected examples of glycomics and its interaction with other omics levels in association with various molecular mechanisms and phenotypes.

\section{Genomics-DNA Level}

Sequence variants could be associated with glycomics at several ways. For example, they could cause gain or loss of a glycosylation site. Prostate cancer risk-associated singlenucleotide polymorphism has been shown to affect prostatespecific antigen (PSA) glycosylation and its function. Functional analysis showed that the missense variant rs61752561 affects PSA stability and structural conformation, and creates an extra glycosylation site (Srinivasan et al., 2019). Moreover, Fan et al. (2018) computationally identified cancer somatic variants causing potential gain or loss of glycosylation.

Beside short variants, structural variants and cytogenetics abnormalities could be associated with glycomics. Glycome expression has been shown to be associated with chromosomal aberrations. Cytogenetic aberrations $\mathrm{t}(11 ; 14), \mathrm{t}(4 ; 14)$, hyperdiploidy, 1q21 gain, and 13q14 deletion have been associated with glycogene expression patterns in multiple myeloma (Moehler et al., 2013). Analysis of genetic variants associated with glycosyltransferases is of special importance since a single glycosyltransferase can glycosylate several proteins. Therefore, a single change in enzyme activity can affect the function of many glycoproteins. Thus, a genetic or epigenetic variant may have a pleiotropic effect on glycosylation, and can affect several downstream pathways and influence the cell metabolism on multiple levels (Vojta et al., 2016).

\section{Transcriptomics-RNA Level}

Several glycomics studies have been performed at the transcriptome level either at a single locus or using a microarray technology in human and animal models in associ- ation with different phenotypes. Mining The Cancer Genome Atlas (TCGA) altered expression of a cancer-associated glycosyltransferase ST6GAL1 was identified in colorectal cancer (CRC) (Venturi et al., 2019). GLYCOv2 glycogene microarray technology was used to identify the differentially expressed glycosylation-related genes in healing mouse corneas (Saravanan et al., 2010).

Of 243 glycan-biosynthesis pathway genes, 60 showed a significantly higher, 20 a lower expression in myeloma compared with normal plasma cell samples (Moehler et al., 2013). Meta-analysis of prostate cancer gene expression data revealed a novel molecular signature, enriched for glycosylation enzymes (Barfeld et al., 2014). Expression of genes that control core fucosylation in hepatocellular carcinoma has been systematically reviewed (Norton and Mehta, 2019). Further systematic reviews and meta-analyses are needed to integrate published data to identify robust expression signatures for development of reliable biomarkers.

\section{Glycoproteomics}

Glycoproteomics is a study of glycoproteins, including their protein identities, sites of glycosylation, and glycan structures. Glycans contribute to protein function by modulating its function through alternative glycosylation; addition of different glycans to the same attachment site on the polypeptide chain (Lauc et al., 2016).

A large number of studies using different methodologies have been published in association with different phenotypes, such as breast, colon, liver, skin, ovary, bladder cancer, and neurological disorders and various structural alterations such as s sialylation, fucosylation, degree of branching, and the expression of specific glycosyltransferases (Azevedo et al., 2017; Christiansen et al., 2014; Palmigiano et al., 2016). For example, cerebrospinal fluid $\mathrm{N}$-glycoproteomics was used for early diagnosis in Alzheimer's disease. The study enabled one 
to assess specific glycosylation patterns in patients and also suggested therapeutics targets, such as glycoenzymes (Palmigiano et al., 2016).

Simultaneous measurement of specific glycoforms combined with protein levels has been suggested to increase the diagnostic potential in pancreatic cancer (Llop et al., 2018). The majority of FDA-approved protein tumor markers currently used in clinical practice are glycoproteins, many of them showing glycosylation alterations in cancer (Lauc et al., 2016). Due to a large number of heterogeneous published results, systematic integrative reviews of the literature are needed to monitor and guide the development of the study field.

\section{Metabolomics}

Metabolomics is a study field that evaluates changes in the metabolome of an organism by exploring the set of small molecules; metabolites. Konjevod et al. (2019) reviewed metabolomic and glycomic findings in post-traumatic stress disorder. The authors suggested integration of these biomarkers with additional omics markers to increase understanding of the biological alterations of this condition.

Wang et al. (2016) performed integrative analysis of proteomic, glycomic, and metabolomic data for liver cancer biomarker discovery. An improved performance was observed by integrative analysis in comparison with separate omics data (Wang et al., 2016). Metabolome and glycome reflect the physiological and pathological conditions in individuals. Some glycans are small molecules, which enables their easy quantification like metabolites (An et al., 2009). A valuable resource for biomarker innovation is the Human Metabolome Database (HMDB), which is the most comprehensive repository of metabolites and could therefore greatly enhance novel biomarker discovery in glycomics (Wishart et al., 2007).

\section{Epigenomics}

DNA methylation, histone tail modifications, and RNAmediated regulation of gene expression are well-known epigenetic mechanisms. Epigenetic modification of genes involved in protein glycosylation has been reported in several studies. However, complex interactions link glycomics and epigenomics. Namely, in contrast with the epigenetic regulation of glycogenes, glycosylation itself epigenetically regulates transcription of other genes. The only form of glycosylation found in the mammalian nucleus, O-GlcNAc, was identified as an epigenetic, histone mark, indicating that glycosylation itself epigenetically regulates gene transcription (Kizuka, 2015).

\section{DNA Methylation}

It has been shown that glycogenes change expression in cancer through aberrant methylation (Vojta et al., 2016). In addition, promoter methylation of the MGAT3 and $B A C H 2$ genes correlates with the composition of the immunoglobulin G glycome in inflammatory bowel disease (Klasić et al., 2018). A complete set of glycogenes could now be screened for their potential regulation by DNA methylation and then if this epigenetic change could be affected with sequence variants.

\section{Histone Modifications}

O-GlcNAc glycosylation (O-GlcNAcylation) is an epigenetic mark, and it was suggested to be pivotal in the modu- lation of chromatin remodeling and in the regulation of gene expression by being part of the histone code (Dehennaut et al., 2014). Covalent post-translational modifications of histones (methylation, acetylation, ubiquitylation, and $\mathrm{O}-$ GlcNAcylation) are dynamically regulated by the interplay of enzyme pairs that add and remove these modifications (Kizuka, 2015).

\section{NcRNomics}

Noncoding RNAs are divided into short $(<200 \mathrm{bp})$ and long noncoding RNAs (lncRNAs) (>200 bp) according to their length. RNA-mediated regulation of gene expression is one of the epigenetics mechanisms, but due to its extent and heterogeneity it is frequently studied as a separate omics type.

\section{MiRNomics}

MicroRNAs (miRNAs) belong to a group of short ncRNAs. Classification of miRNA-related genetic variations is challenging because miRNAs interact with different genomic elements and are studied at different omics levels, including glycomics. MiRNA-associated genetic variability could be studied at miRNA genes and their upstream regulatory elements, genes encoding miRNA silencing machinery (Dicer, Drosha), and miRNA targets (Hrovatin and Kunej, 2018). These categories may also present crossroads for interconnections with glycomics. Mapping post-transcriptional regulation of the human glycome revealed that a subset of miRNAs are involved in regulation of the glycocode (Agrawal et al., 2014) and could therefore be termed glyco-miRNAs.

Glycomic analysis platform, lectin microarray, was used to identify glycosylation signatures in the NCI-60 cell panel. miRNA regulators were then mapped onto genes in glycan biosynthetic pathways that generate the observed glycan structures. Global glycosylation network regulated by miRNAs provides a link between miRNA-mediated control of cell phenotype and the glycome (Agrawal et al., 2014). Beside miRNAs, several other types of short ncRNAs are known, such as siRNAs, snoRNAs, and piRNAs, and should be systematically studied in association with glycomics.

\section{LncRNomics}

lncRNAs have been implicated in a range of developmental processes and diseases; however, they have not yet been systematically studied in association with glycogenes. Most studies focused on selected lncRNAs, such as long intervening/intergenic noncoding RNAs (lincRNAs) and ultraconserved regions (UCRs). For example, lincRNA has been linked with O-glycosylation involved in CRC progression. LINC01296/miR-26a/GALNT3 axis contributes to CRC progression by regulating O-glycosylated MUC1 through PI3K/AKT pathway (Liu et al., 2018). In addition, long noncoding transcript from UCRs, termed transcribedUCRs (T-UCRs), has been shown to reside within $O G T$ gene (Ferdin et al., 2013).

Hypoxia-upregulated T-UCR named HINCUT (hypoxiainduced noncoding ultraconserved transcript) was proposed to be critical for optimal O-GlcNAcylation of proteins in oxygen deprivation in cancer. The current version of the HGNC database includes 438 lncRNAs (https://www.genenames.org/data/ genegroup/\#!/group/788) and could be systematically analyzed for their potential involvement in regulation of glycome. 


\section{Glycolipidomics}

Glycolipidomics is a study field focused on identification and quantification of glycolipids. Glycolipids have potential as specific biomarkers for various physiological and pathological processes. They have been shown to be involved in development of neurological and neurodegenerative diseases, such as Parkinson's disease, Alzheimer's disease, Lewy body dementia, and frontotemporal dementia. In addition, cancer-associated glycosphingolipids have been used as markers for diagnosis and targets for immunotherapy of malignant tumors (Furukawa et al., 2019). In addition, cancer-associated glycosphingolipids have been used as markers for diagnosis and targets for immunotherapy of malignant tumors (Furukawa et al., 2019). Recent advancements in glycolipid biomarker discovery by mass spectrometry are reviewed in Dehelean et al. (2019).

\section{Interactomics}

Interactome is a whole set of molecular interactions in cell including glycan interactions. Binding between glycans and glycan-binding proteins (GBPs) was shown to be involved in almost all physiological and pathophysiological processes, including cell recognition and signaling, immune response, infections, cancer, and neurodegenerative disorders. These interactions could now be studied using high-throughput techniques (Kitov et al., 2019). Lectins are GBPs and are cataloged in the UniLectin3D a database with curated information on 3D structures and interacting ligands (Bonnardel et al., 2019). The interactions between lectins and glycans on plasma membranes have emerged as important regulatory factors in corneal biology and pathophysiology, including corneal infection and inflammation (AbuSamra and Argüeso, 2018).

The complete protein-glycan interactions (protein-glycan interactome) have not yet been determined or estimated (Cummings and Pierce, 2014). An estimation of the number of these interactions is an important study question for future studies. Bioinformatics tool GenProBiS (Konc et al., 2017), for example, enables protein-protein, protein-nucleic acid, and protein-compound binding site analysis, including glycosylation and other post-translational modification sites. Moreover, it maps sequence variants to protein structures. Further development of advanced bioinformatics tools enabling big data analyses will enable prioritization of the targets for experimental validation and substantially contribute to the development of the interatomics field.

\section{Other Omics Types}

Several other omics types need to be systematically reviewed in association with glycomics in future studies, for example, regulomics and comparative genomics. Glycomics is involved in a complex interplay of molecular interactions; however, little is known about the regulatory networks controlling these complex processes. Transfer of glycomics knowledge gained from animal models (comparative glycomics) is also one of the research directions.

Beside basic (classic) omics types, omics applications need to be reviewed, such as pharmacogenomics, environmental omics, nutrigenomics, and iatromics. Glycans have a large potential for therapeutics as many recombinant pharmaceuticals, like thera- peutic monoclonal antibodies, are glycoproteins (Lauc et al., 2016). In addition, $O$-GlcNAcylation is a post-translational modification that links nutrition to epigenetics. Uridine diphosphate-GlcNAc (UDP-GlcNAc) and $O$-GlcNAcylation are therefore considered as sensors of the nutritional state of the organism (Dehennaut et al., 2014).

\section{Multiomics}

The glycomics study field has shifted from single locus to whole genome-level study approaches. Moreover, it has shifted from single omics to integrated omics understanding of disease mechanisms. Integration of the transcriptome and glycome was performed for identification of glycan cell signatures (Bennun et al., 2013). A number of studies with integration of multiple platforms are expected in the near future as they are increasingly produced. The number of included omics levels into integrative analyses is expected to increase as well. Data from 13 omics layers including glycomics were synthesized with the aim to enable a step toward a systems view on pathological mechanisms and for prioritization of novel potential biomarkers in Parkinson's disease and asthma (Pecak et al., 2018; Redenšek et al., 2018).

Mass spectrometry imaging has emerged as a powerful tool for the detection of various molecules, including proteins, peptides, glycans, metabolites, phospholipids, and glycolipids, in tissue samples, and therefore presents an important tool for multiomics studies ( $\mathrm{Xu}$ and $\mathrm{Li}, 2019)$. The integration of multiple data sets from different omics levels represents an important application of systems biology for understanding complex cellular processes involved in glycobiology.

\section{Bioinformatics Tools and Databases}

Glycoinformatics is a field of informatics that studies glycome. Bioinformatics tools are now needed to collect glycomics data and enable the user to search, display, and connect with other sources of related proteomics, genomics, and interactomics information. GlyConnect (https://glyconnect .expasy.org/) is the central platform for glycoinformatics. It has been developed to gather, monitor, integrate, and visualize glycomics data and to integrate multiple data types (Alocci et al., 2019). Major web-based glycan resources are reviewed in Bennun et al. (2016) and include the following: GlycomeDB, GlycoSuiteDB, Japan Consortium for Glycobiology and Glycotechnology Database (JCGGDB), and Complex Carbohydrate Structure Database (CCSD; CarbBank).

The current version of the Nucleic Acids Research (NAR) database lists 14 resources under the "Carbohydrates" subsection https://www.oxfordjournals.org/nar/database/subcat/ 4/12 (Rigden and Fernández, 2019) and presents a starting point for glycomics research. To facilitate the data visualization and visual comparison of glycan array data, GLAD (GLycan Array Dashboard) tool has been developed, which enables one to visualize and mine glycan array data (Mehta and Cummings, 2019).

\section{International Collaborative Initiatives}

International collaborative initiatives have already contributed to development of resources in technologies in glycomics such as Consortium for Functional Glycomics (CFG) and the National Center for Functional Glycomics 
(NCFG). Data for $>3900$ experiments have been made public by the CFG (www.functionalglycomics.org/) (Mehta and Cummings, 2019). The Human Glycome Project (HGP) https://human-glycome.org/ supports individual researchers by sharing resources and experiences, as well as coordinating research activities on a global level.

\section{Challenges and Future Directions}

Despite several recent advancements in the glycomics field, a systematic map of all possible connections between glycomics and other omics types has not yet been developed. Glycomics is involved in a complex interplay of molecular interactions; however, little is known about the regulatory networks controlling these processes. On the contrary, several physiological and pathological mechanisms are known, but the knowledge is scattered across various publications and databases, and therefore does not enable a holistic view of this study field. Therefore, researchers are not always aware that the analyzed genetic locus is linked with glycans and that a result of the study is interconnected with glycomics level. Integrating glycomics with other omics levels is a challenge at one locus, and even larger task at the genomewide level.

Importantly, the present literature analysis also revealed several salient knowledge gaps and future directions in the glycomics field. I highlight them below for future consideration of scholars in the field of glycomics specifically, and omics system sciences broadly:

(1) Development of a detailed map of all connections between glycomics and other omics types, such as epigenomics, miRNomics, proteomics, lipidomics, and interactomics

(2) Systematic analysis and prioritization of all known sequence variants, associated with glycomics, for example, located within genes encoding for glycosyltransferases and glycosidases or located within glyco-miRNA target sites

(3) Harnessing or finding novel utilities for heterogeneous published data and their integration with glycomics

(4) Development of multiplatform approach including high-throughput $N$-glycan analysis techniques

(5) Development of multiomics biomarker panels, including glycans

(6) Further extending of systems biology with systems glycobiology and systems glycopathology

(7) Integration of personalized glycomedicine into personalized medicine

(8) Hands-on laboratory and bioinformatics training in the methods of glycomics

(9) Development of an initiative for reporting standardization in glycomics

(10) Performing systematic reviews and meta-analyses to integrate published data to identify potential new biomarkers, to identify knowledge gaps and guidelines for future studies.

\section{Conclusions}

Glycomics is a very broad study field and interacts with several omics types. However, it is only one of the omics levels of a complex regulatory interplay, consisting of a large number of genomics elements. Further development of highthroughput analysis techniques and bioinformatics tools is expected in the future and will enable to integrate all available glycomics data into a single map, which will enable biomarker development and identification of potential new therapeutic targets.

The present review presents only selected examples how glycomics overlaps with other omics types and presents a framework for further systematic reviews. Integration of all published data related to glycomics is very extensive for one research group. Therefore, a consortium leading the community such as HGP is of importance to organize the development of the complete glycomics resource, and to coordinate planning of research of glycobiology and personalized glycomedicine. Research developments indicate that glycomics should be included in an integrative multiomics approach toward development of robust disease biomarkers enabling patient-tailored clinical decisions.

\section{Author Disclosure Statement}

The author declares that no conflicting financial interests exist.

\section{Funding Information}

This work was supported by the Slovenian Research Agency (ARRS) through the Research program P4-0220.

\section{References}

Abusamra DB, and Argüeso P. (2018). Lectin-glycan interactions in corneal infection and inflammation. Front Immunol 9, 2338.

Adua E, Russell A, Roberts P, Wang Y, Song M, and Wang W. (2017). Innovation analysis on postgenomic biomarkers: Glycomics for chronic diseases. OMICS 21, 183-196.

Agarwal V, Bell GW, Nam JW, and Bartel DP. (2015). Predicting effective microRNA target sites in mammalian mRNAs. Elife 4, DOI: 10.7554/eLife.05005.

Agrawal P, Kurcon T, Pilobello KT, et al. (2014). Mapping posttranscriptional regulation of the human glycome uncovers microRNA defining the glycocode. Proc Natl Acad Sci U S A 111, 4338-4343.

Alocci D, Mariethoz J, Gastaldello A, et al. (2019). GlyConnect: Glycoproteomics goes visual, interactive, and analytical. J Proteome Res 18, 664-677.

An HJ, Kronewitter SR, De Leoz ML, and Lebrilla CB. (2009). Glycomics and disease markers. Curr Opin Chem Biol 13, 601-607.

Azevedo R, Peixoto A, Gaiteiro C, et al. (2017). Over forty years of bladder cancer glycobiology: Where do glycans stand facing precision oncology? Oncotarget 8, 9173491764.

Barfeld SJ, East P, Zuber V, and Mills IG. (2014). Metaanalysis of prostate cancer gene expression data identifies a novel discriminatory signature enriched for glycosylating enzymes. BMC Med Genomics 7, 513.

Bennun SV, Hizal DB, Heffner K, Can O, Zhang H, and Betenbaugh MJ. (2016). Systems glycobiology: Integrating glycogenomics, glycoproteomics, glycomics, and other 'omics data sets to characterize cellular glycosylation processes. J Mol Biol 428, 3337-3352. 
Bennun SV, Yarema KJ, Betenbaugh MJ, and Krambeck FJ. (2013). Integration of the transcriptome and glycome for identification of glycan cell signatures. PLoS Comput Biol 9, e1002813.

Bonnardel F, Mariethoz J, Salentin S, et al. (2019). UniLectin3D, a database of carbohydrate binding proteins with curated information on 3D structures and interacting ligands. Nucleic Acids Res 47, D1236-D1244.

Christiansen MN, Chik J, Lee L, Anugraham M, Abrahams JL. and Packer NH. (2014). Cell surface protein glycosylation in cancer. Proteomics 14, 525-546.

Cummings RD, and Pierce JM. (2014). The challenge and promise of glycomics. Chem Biol 21, 1-15.

Dehelean L, Sarbu M, Petrut A, and Zamfir AD. (2019). Trends in glycolipid biomarker discovery in neurodegenerative disorders by mass spectrometry. Adv Exp Med Biol 1140, 703-729.

Dehennaut V, Leprince D, and Lefebvre T. (2014). OGlcNAcylation, an epigenetic mark. Focus on the histone code, TET family proteins, and polycomb group proteins. Front Endocrinol (Lausanne) 5, 155.

Fan Y, Hu Y, Yan C, et al. (2018). Loss and gain of N-linked glycosylation sequons due to single-nucleotide variation in cancer. Sci Rep 8, 4322.

Ferdin J, Nishida N, Wu X, et al. (2013). HINCUTs in cancer: Hypoxia-induced noncoding ultraconserved transcripts. Cell Death Differ 20, 1675-1687.

Furukawa K, Ohmi Y, Ohkawa Y, et al. (2019). New era of research on cancer-associated glycosphingolipids. Cancer Sci 110, 1544-1551.

Gabius HJ, André S, Jiménez-Barbero J, Romero A, and Solís D. (2011). From lectin structure to functional glycomics: Principles of the sugar code. Trends Biochem Sci 36, 298313.

Gabius HJ, and Kayser K. (2014). Introduction to glycopathology: The concept, the tools and the perspectives. Diagn Pathol 9, 4.

Geissner A, and Seeberger PH. (2016). Glycan arrays: From basic biochemical research to bioanalytical and biomedical applications. Annu Rev Anal Chem (Palo Alto Calif) 9, 223-247.

Hart GW, and Copeland RJ. (2010). Glycomics hits the big time. Cell 143, 672-676.

Hrovatin K, and Kunej T. (2018). Classification of miRNArelated sequence variations. Epigenomics 10, 463-481.

Kitov PI, Kitova EN, Han L, et al. (2019). A quantitative, highthroughput method identifies protein-glycan interactions via mass spectrometry. Commun Biol 2, 268.

Kizuka Y. (2015). Epigenetic regulation of and by glycosylation. In: Glycoscience: Biology and Medicine. Taniguchi N, Endo T, Hart GW, Seeberger PH, and Wong C-H, eds. Tokyo: Springer Japan, 1129-1134.

Klasić M, Markulin D, Vojta A, et al. (2018). Promoter methylation of the MGAT3 and $B A C H 2$ genes correlates with the composition of the immunoglobulin $\mathrm{G}$ glycome in inflammatory bowel disease. Clin Epigenetics 10, 75.

Konc J, Skrlj B, Erzen N, Kunej T, and Janezic D. (2017). GenProBiS: Web server for mapping of sequence variants to protein binding sites. Nucleic Acids Res 45, W253W259.

Konjevod M, Tudor L, Svob Strac D, et al. (2019). Metabolomic and glycomic findings in posttraumatic stress disorder. Prog Neuropsychopharmacol Biol Psychiatry 88, 181-193.
Lauc G, Pezer M, Rudan I, and Campbell H. (2016). Mechanisms of disease: The human N-glycome. Biochim Biophys Acta 1860, 1574-1582.

Liu B, Pan S, Xiao Y, Liu Q, Xu J, and Jia L. (2018). LINC01296/miR-26a/GALNT3 axis contributes to colorectal cancer progression by regulating O-glycosylated MUC1 via PI3K/AKT pathway. J Exp Clin Cancer Res 37, 316.

Llop E, E Guerrero P, Duran A, et al. (2018). Glycoprotein biomarkers for the detection of pancreatic ductal adenocarcinoma. World J Gastroenterol 24, 2537-2554.

Ly M, Laremore TN, and Linhardt RJ. (2010). Proteoglycomics: Recent progress and future challenges. OMICS 14, 389-399.

Mehta AY, and Cummings RD. (2019). GLAD: GLycan array dashboard, a visual analytics tool for glycan microarrays. Bioinformatics 35, 3536-3537.

Moehler TM, Seckinger A, Hose D, et al. (2013). The glycome of normal and malignant plasma cells. PLoS One 8, e83719.

Murphy K, Murphy BT, Boyce S, et al. (2018). Integrating biomarkers across omic platforms: An approach to improve stratification of patients with indolent and aggressive prostate cancer. Mol Oncol 12, 1513-1525.

Narimatsu Y, Joshi HJ, Nason R, et al. (2019). An atlas of human glycosylation pathways enables display of the human glycome by gene engineered cells. Mol Cell 75, 394407.e5.

Norton PA, and Mehta AS. (2019). Expression of genes that control core fucosylation in hepatocellular carcinoma: Systematic review. World J Gastroenterol 25, 2947-2960.

Palmigiano A, Barone R, Sturiale L, et al. (2016). CSF Nglycoproteomics for early diagnosis in Alzheimer's disease. J Proteomics 131, 29-37.

Pecak M, Korošec P, and Kunej T. (2018). Multiomics data triangulation for asthma candidate biomarkers and precision medicine. OMICS 22, 392-409.

Pirih N, and Kunej T. (2017). Toward a taxonomy for multiomics science? Terminology development for whole genome study approaches by omics technology and hierarchy. OMICS 21, 1-16.

Pirih N, and Kunej T. (2018). An updated taxonomy and a graphical summary tool for optimal classification and comprehension of omics research. OMICS 22, 337-353.

Redenšek S, Dolžan V, and Kunej T. (2018). From genomics to omics landscapes of parkinson's disease: Revealing the molecular mechanisms. OMICS 22, 1-16.

Reily C, Stewart TJ, Renfrow MB, and Novak J. (2019). Glycosylation in health and disease. Nat Rev Nephrol 15, 346366.

Rigden DJ, and Fernández XM. (2019). The 26th annual nucleic acids research database issue and molecular biology database collection. Nucleic Acids Res 47, D1-D7.

Saravanan C, Cao Z, Head SR, and Panjwani N. (2010). Analysis of differential expression of glycosyltransferases in healing corneas by glycogene microarrays. Glycobiology 20, 13-23.

Smith DF, Cummings RD, and Song X. (2019). History and future of shotgun glycomics. Biochem Soc Trans 47, 1-11.

Srinivasan S, Stephens C, Wilson E, et al. (2019). Prostate cancer risk-associated single-nucleotide polymorphism affects prostate-specific antigen glycosylation and its function. Clin Chem 65, e1-e9.

Varki A. (2017). Biological roles of glycans. Glycobiology 27, $3-49$. 
Venturi G, Ferreira IG, Pucci M, et al. (2019). Impact of sialyltransferase ST6GAL1 overexpression on different colon cancer cell types. Glycobiology, [Epub ahead of print]; DOI: 10.1093/glycob/cwz053.

Vojta A, Samaržija I, Bočkor L, and Zoldoš V. (2016). Glycogenes change expression in cancer through aberrant methylation. Biochim Biophys Acta 1860, 1776-1785.

Wang M, Yu G, and Ressom HW. (2016). Integrative analysis of proteomic, glycomic, and metabolomic data for biomarker discovery. IEEE J Biomed Health Inform 20, 12251231.

Wishart DS, Tzur D, Knox C, et al. (2007). HMDB: The human metabolome database. Nucleic Acids Res 35, D521D526.

$\mathrm{Xu} \mathrm{G}$, and Li J. (2019). Recent advances in mass spectrometry imaging for multiomics application in neurology. J Comp Neurol 527, 2158-2169.

Yates B, Braschi B, Gray KA, Seal RL, Tweedie S, and Bruford EA. (2017). Genenames.org: The HGNC and VGNC resources in 2017. Nucleic Acids Res 45, D619-D625.

Address correspondence to:

Tanja Kunej, PhD

Department of Animal Science

Biotechnical Faculty

University of Ljubljana

Groblje 3

Domzale SI-1230

Slovenia

E-mail: tanja.kunej@bf.uni-lj.si

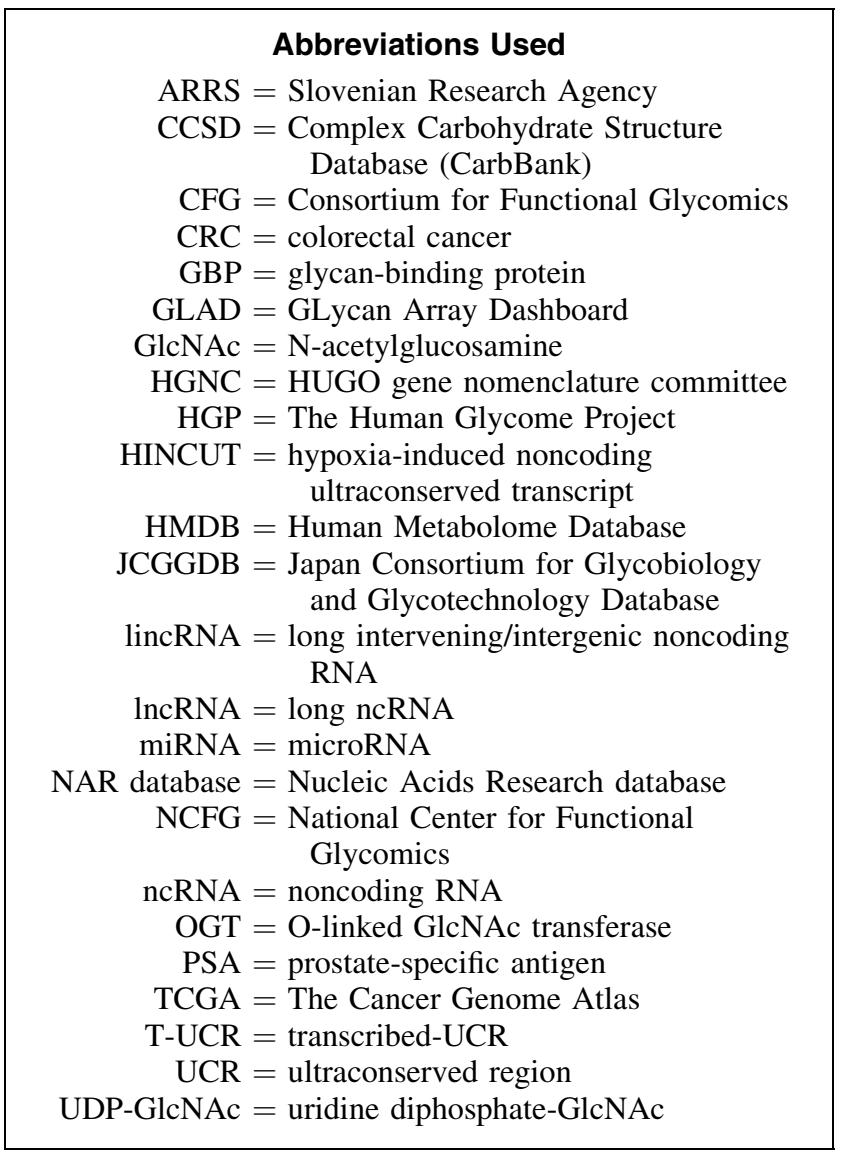

